\title{
BMJ Global Health Efficacy of a bovine colostrum and egg- based intervention in acute childhood diarrhoea in Guatemala: a randomised, double-blind, placebo-controlled trial
}

\author{
James T Gaensbauer, ${ }^{1,2}$ Mario A Melgar, ${ }^{3}$ Diva M Calvimontes, ${ }^{3}$ Molly M Lamb,,${ }^{1,2}$ \\ Edwin J Asturias, ${ }^{1,2}$ Ingrid L Contreras-Roldan, ${ }^{4}$ Samuel R Dominguez, ${ }^{5}$ \\ Christine C Robinson, ${ }^{6}$ Stephen Berman ${ }^{1,2}$
}

To cite: Gaensbauer JT, Melgar MA, Calvimontes DM, et al. Efficacy of a bovine colostrum and egg-based intervention in acute childhood diarrhoea in Guatemala: a randomised, doubleblind, placebo-controlled trial. BMJ Glob Health 2017;2:e000452. doi:10.1136/ bmjgh-2017-000452

Handling editor Alberto GarciaBasteiro

- Additional material is published online only. To view, please visit the journal online (http://dx.doi.org/10.1136/ bmjgh-2017-000452).

Findings from this study were presented at the Annual Meeting of the American Society for Tropical Medicine and Hygiene, Atlanta, USA. October, 2016.

Received 29 June 2017 Revised 20 0ctober 2017 Accepted 25 0ctober 2017

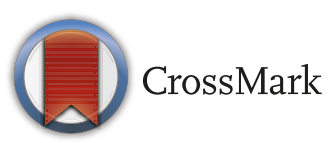

For numbered affiliations see end of article.

Correspondence to Dr James T Gaensbauer; james.gaensbauer@ childrenscolorado.org

\section{ABSTRACT}

Background Treatments for paediatric diarrhoeal disease are limited. We assessed the impact of a bovine colostrum and egg-based treatment designed to reduce diarrhoea duration through non-specific and pathogendirected mechanisms in children.

Methods Randomised, double-blind, placebo-controlled trial of PTM202, derived from bovine colostrum and hyperimmune hen's egg on the duration of acute diarrhoeal disease in Guatemalan children. PTM202 contains specific immunoglobulins that target rotavirus, enterotoxigenic Escherichia coli, Shiga toxin-producing E. coli and Salmonella. Children aged 6-35 months presenting to three sites (one rural and two urban) with acute non-bloody diarrhoea were computer randomised to receive three daily doses of PTM202 or placebo. The primary outcome was the post-treatment duration of diarrhoea assessed in the per protocol population. Diarrhoeal pathogens were identified in stool by multiplex PCR (FilmArray Gastrointestinal-Panel, BioFire, Salt Lake City, Utah, USA). Key secondary outcomes included postdiarrhoeal weight gain and impact on diarrhoeal duration stratified by study site and presence of PTM202-targeted organisms in stool at enrolment. Safety was assessed in all participants.

Results From 9 March 2015 to 25 January 2016, 325 children were enrolled, and 301 (154 intervention and 147 placebo) were analysed for the primary outcome. No difference in diarrhoea duration was observed between intervention and placebo in the total population, but a significant reduction was observed in the treatment group among children with at least one targeted pathogen in stool $(H R=1.46, P=0.02)$, an effect most pronounced in urban subjects (HR 2.20, $\mathrm{P}=0.007$ ) who had fewer stool pathogens and better nutritional status. No impact on 2-week or 4-week weight gain was noted. No adverse events attributed to PTM202 occurred.

Conclusion Results demonstrate the potential to target specific pathogens occurring in children with acute nonbloody diarrhoea and shorten illness duration using a novel, safe, nutrition-based intervention. PTM202 may represent a new tool to ameliorate the effects of acute diarrhoeal disease in low/middle-income populations. Trial registration number NCT02385773; Results.

\section{INTRODUCTION}

Diarrhoeal disease was the cause of approximately $11 \%$ of all childhood deaths between the years 2000 and 2011 and is the fourth most common cause of global childhood morbidity, with an estimated 1.7 billion episodes per year in children aged less than 5 years. ${ }^{12}$ Furthermore, diarrhoea has an additive negative effect on children already affected by poor nutrition. Acute episodes are associated with dehydration, electrolyte and micronutrient loss, poor intestinal healing and weight loss. Delayed return to normal bowel function can impair nutritional recovery in vulnerable children and contribute to both short-term weight faltering and potentially long-term malnutrition, growth failure and increased mortality. ${ }^{34}$

Current therapies for the treatment of acute diarrhoeal disease in children in low/ middle-income countries (LMICs) are limited. Treatment is focused primarily around provision of oral rehydration solution (ORS), which has resulted in significant increases in survival since its introduction. ${ }^{5}$ However, ORS does not shorten the duration of acute diarrhoeal episodes, nor does it promote healing of inflamed intestinal mucosa or provide nutritional rehabilitation. Supplementation of zinc, a micronutrient often deficient in low-income paediatric populations that plays an important role in mucosal integrity, has been shown to reduce the duration of acute diarrhoeal episodes and prevent future occurrences, but questions remain about efficacy in infants younger than 6 months and in children without underlying zinc deficiency. ${ }^{6}$ Antibiotics may benefit some types of bacterial acute infectious diarrhoea but have no effect on viral etiologies. Treatment may 


\section{Key questions}

\section{What is already known about this topic?}

- Diarrhoeal disease is a major cause of global childhood morbidity and mortality, and the burden of disease falls primarily in children in low/middle-income countries.

- Oral rehydration therapy prevents dehydration and reduces morbidity and mortality from acute paediatric diarrhoeal disease but does not alter the duration or severity of disease episodes.

- Evidence from prior clinical trials suggests that nutrition-based therapies, including bovine colostrum and chicken egg containing pathogen-specific $\lg \bigotimes$, may ameliorate diarrhoeal illness, but studies are limited by low numbers of subjects, heterogeneous study populations and a focus on single diarrhoeal pathogens.

\section{What are the new findings?}

- PTM202 is a novel and innovative nutrition-based diarrhoeal treatment incorporating bovine colostrum and hen's egg, which by design specifically targets rotavirus, enterotoxigenic Escherichia coli, Shiga toxin-positive E. coli and salmonella, and has in vitro-neutralising cross-reactivity against two other pathogens: Shigella and norovirus.

- In this randomised, double-blind, placebo-controlled trial of Guatemalan children presenting with acute non-bloody diarrhoea, administration of PTM202 one time per day for 3 days led to a reduction in diarrhoeal duration among children whose diarrhoeal stool at enrolment contained one or more PTM202-targeted organism, as determined by multiplex PCR.

- Treatment was safe and well tolerated.

\section{Recommendations for policy}

- Demonstration that PTM202 can simultaneously target multiple stool pathogens and reduce diarrhoeal duration suggests that the treatment adds to the currently limited therapeutic armamentarium against one of the most significant global causes of childhood disease.

- The efficacy of the PTM202 was greatest among children living in an urban setting, who had better nutritional status and fewer coincident non-targeted pathogens.

- The concepts demonstrated in this trial suggest the potential for future developments to target additional pathogens and broaden the population for whom this treatment may benefit.

promote complications from certain bacterial agents, and antibiotic overuse contributes to the global crisis of antimicrobial resistance. ${ }^{7}$ Probiotic treatment has been shown to decrease severity and duration of diarrhoea in children, particularly with watery diarrhoea resulting from rotavirus, but use of probiotics for paediatric diarrhoea in the developing world is limited by cost. ${ }^{89}$

Nutritional-based interventions represent a novel area of potential diarrhoea therapeutics. Breast milk contains both pathogen-specific antibody (primarily $\operatorname{IgA}$ ) and numerous gut-active compounds that may promote intestinal healing, and breastfeeding through diarrhoeal illness is strongly recommended by the WHO. As breast milk is not available to all children, one strategy to mimic its beneficial composition is the use of bovine colostrum, which has been variably effective in the treatment of childhood diarrhoea. ${ }^{10}$ Another source of nutrition-based diarrhoeal treatment is hen's egg, which contains IgY, the specificity of which can be enhanced through hen vaccination against individual human diarrhoeal pathogens. ${ }^{11}$

PTM202 is derived from both bovine colostrum and egg from hens that receive vaccines approved by the USDA for use in production animals. By design, it combines colostrum and egg solids to deliver specific IgG and IgY as well as non-antibody-related products (eg, lactoferrin, arginine and glutamine). PTM202 specifically targets rotavirus, enterotoxigenic Escherichia coli (ETEC), Shiga toxin-positive E. coli (STEC) and salmonella (directly targeted) and has in vitro-neutralising cross-reactivity against two other pathogens: Shigella/ enteroinvasive E. coli (EIEC) and norovirus (indirectly targeted).

To assess the impact of PTM202 on recovery from an acute episode of diarrhoea, we undertook a randomised, double-blind, placebo-controlled trial in Guatemalan children. The study was designed to evaluate the impact of PTM202 on time to resolution of acute non-bloody diarrhoeal illness and postdiarrhoeal weight recovery. Given the potential to target specific diarrhoeal pathogens with PTM202, we aimed additionally to assess the impact of PTM202 on diarrhoeal duration in subjects with PTM202-targeted organisms in stool at the time of enrolment.

\section{METHODS \\ Study design}

This randomised, double-blind, placebo-controlled trial was conducted at three investigational sites in Guatemala: the Hospital Roosevelt Department of Paediatrics in Guatemala City, the Hospital Infantil de Infectologia y Rehabilitacion in Guatemala City and the Clinic of the Centre for Human Development, in the rural southwest lowlands of Guatemala, in association with the Centre for Global Health, Colorado School of Public Health, Aurora, Colorado. Ethical approval was obtained from the Colorado Multiple Institutional Review Board and in Guatemala from the National Committee for Ethics in Health, Ministry of Public Health and Social Assistance, Hospital Roosevelt Ethics Committee and the Universidad del Valle de Guatemala Ethics Committee. All trial procedures were designed and implemented according to the principles embodied in the Declaration of Helsinki. Subjects who are 6 to 35 months of age with severe or moderate diarrhoea were enrolled based on diarrhoeal severity in two parallel arms with an initial enrolment target of 300 subjects in each arm. Inclusion criteria for both arms included any gender child with acute diarrhoea of $<72$ hours duration and more than three liquid stools in the previous 24 hours. One of the criteria for severe arm inclusion was an anticipated or established requirement for $>8$ hours of supervised oral or intravenous inpatient rehydration in hospital facilities. Moderate arm inclusion was an anticipated need for less 
than 8 hours of rehydration. Exclusion criteria included any clinical condition for which oral intake of intervention or placebo was contraindicated in the opinion of the attending physician, a history of hypersensitivity or adverse reaction to milk or egg product, condition improving in last 24 hours per parental report, mild disease severity (no recommended period of observed rehydration therapy per attending physician), bloody diarrhoea at presentation, severe malnutrition (defined as weight-for-height z-score $<-3$ from WHO-published standards), major congenital defects or serious chronic illness, receipt of probiotics in 72 hours prior to enrolment or recommended treatment with probiotics by the attending physician or any confirmed or suspected immunosuppressive or immunodeficient condition. Parents or guardians of all subjects provided written informed consent prior to enrolment.

\section{Randomization and masking}

Randomization of subjects within both arms occurred at the initial study visit following informed consent, assessment of inclusion and exclusion criteria and obtainment of baseline data, using a computer-generated randomization schedule designed by a statistician who played no further role in the trial. Subjects were allocated sequentially from this list at the time of enrolment. All study personnel, including investigators, study nurses, data managers and lab personnel, as well as parents/ legal guardians, remained blinded until all study procedures were complete for all subjects, and all data were completed and certified. Study intervention and placebo (both dry powders) were matched for consistency, taste and smell when reconstituted with water and packaged at a separate facility in to sealed, opaque envelopes imprinted with the randomization number.

\section{Procedures}

The study nutritional intervention, PTM202 (PanTheryx, Inc., Boulder, USA), is a dry powder for reconstitution, comprised of a proprietary mixture of dried bovine colostrum and dried whole egg. Each sachet contains $7 \mathrm{~g}$ of dry powder and is reconstituted in $30 \mathrm{~mL}$ of water immediately prior to administration. Treatment was administered as one full reconstituted sachet by mouth one time per day for 3 days for a total of three sachets. There was no alteration in dose based on age or weight of the subject. The placebo was Enfamil PurAmino, a hypoallergenic amino acid-based infant formula for the dietary management of infants and toddlers with severe cow's milk protein allergy, reconstituted by study nurses in water and administered in equal volume and frequency to the study intervention. Per protocol, ongoing participation in the trial required ingestion of a minimum of two doses of study intervention or placebo.

Study procedures required five study visits (on days 1, 2, 3,17 and 31) and two scheduled phone assessments (days 10 and 24). Patients were either seen daily in hospital if admitted or returned to the study site if outpatient.
Treatment with PTM202 or placebo was administered on days 1, 2 and 3. Clinical assessments and weights were obtained at all in-person study visits. Stool samples were collected on day 1 for all subjects and day $31( \pm 3)$. For subjects who had diarrhoea persisting after study day 3 , an additional phone contact was made daily starting on day 4 until the resolution of diarrhoea was confirmed. Non-programmed visits and contacts, adverse events and protocol deviations were documented throughout the study period.

\section{Laboratory procedures}

On study days 1 and 31 , a $5-15 \mathrm{~mL}$ of stool sample was collected. Day 1 sample was collected by study nurses, and day 31 sample was collected at home by parents within 24 hours of the visit. Samples in the urban site were stored initially at $-20^{\circ} \mathrm{C}$ and transported daily on wet ice to a central laboratory in Guatemala City where they were immediately processed. In the rural site, samples were frozen at $-80^{\circ} \mathrm{C}$ on site and transported two times per month on dry ice to the central laboratory. At the central laboratory, specimens were aliquoted into multiple cryovials (without melting previously frozen rural samples) and stored at $-80^{\circ} \mathrm{C}$. The raw samples were shipped on dry-ice by air courier to the Microbiology Laboratory at Children's Hospital Colorado, Aurora, USA, and stored at $-80^{\circ} \mathrm{C}$ until tested. After thawing, $1 \mathrm{~mL}$ of each stool was placed into a phial containing $3 \mathrm{~mL}$ of Para-Pack C\&S stool transport medium (Meridian Bioscience, Cincinnati, Ohio, USA), mixed well and tested for stool pathogens by a multiplex PCR assay (FilmArray GI-Panel; BioFire, Salt Lake City, Utah, USA) according to the package insert. This assay simultaneously detects 22 human diarrhoeal pathogens (see table 4), including bacteria, viruses and protozoa.

\section{Outcomes}

The primary outcome was postintervention duration of diarrhoea, measured as the time in hours from the first dose of treatment/placebo until a time point defined by the last diarrhoeal stool prior to passage of a formed stool or no stool output for 12 hours. Secondary outcomes included assessment of weight change between study day 3 (rehydrated weight) and time points 2 and 4 weeks later. Additional analyses of these outcomes were stratified by urban or rural site, diarrhoea severity defined by estimated per cent dehydration at enrolment (within each arm) and presence of diarrhoeal pathogens targeted directly and/or indirectly by PTM202 at the time of enrolment.

Adverse events were solicited from all subjects using weekly diary cards for the duration of the 1-month study period. Unsolicited adverse events were captured from medical records and direct communication with subjects and medical providers. All serious adverse events (SAEs) and suspected unexpected serious adverse reactions were reported within 24 hours to the principal investigator and ethics committees for review. 


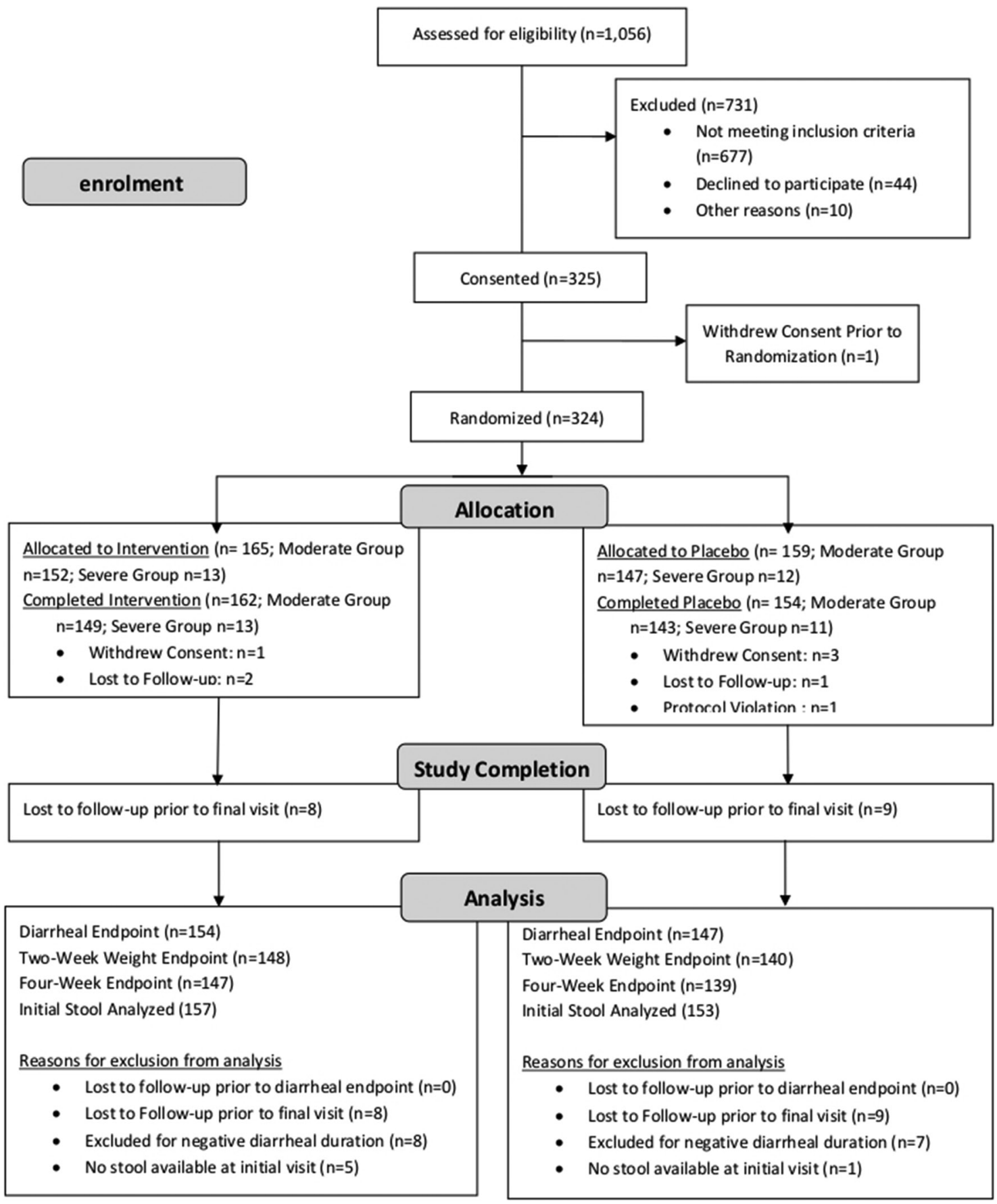

Figure 1 CONSORT trial profile and end point achievement.

\section{Statistical analysis}

Sample size calculations were performed a priori for both the primary outcome of diarrhoea duration and the secondary outcome of weight gain at 14 days postrehydration. Calculations used assumptions about diarrhoea duration and weight gain postdiarrhoea from published literature. ${ }^{1213}$ A sample size of 300 had a power of $82 \%$ (two sample comparison of means, two-sided 0.05 significance) to detect a $10 \%$ reduction in diarrhoea duration, assuming a mean placebo diarrhoea duration of 104 hours with an SD of 28 hours, and $80 \%$ power to detect a difference of $0.20 \mathrm{~kg}$ in the weight change between the groups at 2 weeks assuming a $3 \%$ postrehydration weight gain in the placebo group and an SD of $0.44 \mathrm{~kg}$.

Participants were included in the analysis of primary and secondary outcomes per protocol. Safety analysis was conducted for subjects who ingested any amount of study intervention. Due to low enrolment number in the severe arm, the results were combined with those of children with moderate diarrhoea for analysis. Baseline demographics were compared between urban and rural subjects using t-tests for continuous variables and $\chi^{2}$ tests for categorical variables. Median diarrhoeal durations were compared using log-rank tests, and Kaplan-Meier curves were 
Table 1 Baseline characteristics for the combined moderate and severe groups enrolled at urban and rural sites, by intervention arm

\begin{tabular}{|c|c|c|c|c|}
\hline \multirow[b]{2}{*}{ Variable } & \multicolumn{2}{|c|}{ Urban $(n=144)$} & \multicolumn{2}{|c|}{ Rural ( $n=172)$} \\
\hline & PTM202 & Placebo & PTM202 & Placebo \\
\hline Age (months) ${ }^{*}$ & $17.2(7.1)$ & $15.7(6.4)$ & $19.0(8.0)$ & $18.4(7.6)$ \\
\hline Weight-for-age WHO z-score at enrolment ${ }^{\star}$ & $-0.7(0.9)$ & $-0.9(1.0)$ & $-1.6(1.1)$ & $-1.7(1.1)$ \\
\hline Weight for Length WHO z-score at enrolment* & $-0.6(1.0)$ & $-0.8(1.0)$ & $-1.1(1.0)$ & $-1.3(1.1)$ \\
\hline Antibiotics prescribed at initial visit & $10(13.2 \%)$ & $6(8.8 \%)$ & $15(17.4 \%)$ & $9(10.5 \%)$ \\
\hline Zinc prescribed at initial visit & $30(39.5 \%)$ & $29(42.7 \%)$ & $54(62.8 \%)$ & $57(66.3 \%)$ \\
\hline \multicolumn{5}{|l|}{ Sext } \\
\hline Female & $33(43.4 \%)$ & $32(47.1 \%)$ & $47(54.7 \%)$ & $35(40.7 \%)$ \\
\hline Male & $43(56.6 \%)$ & $36(52.9 \%)$ & $39(45.4 \%)$ & $51(59.3 \%)$ \\
\hline \multicolumn{5}{|l|}{ Water supply to house } \\
\hline Public or bottled water & $70(92.1 \%)$ & 65 (95.6\%) & $13(15.1 \%)$ & $6(7.0 \%)$ \\
\hline Rainwater or water tank & $3(4.0 \%)$ & $3(4.4 \%)$ & $1(1.2 \%)$ & $0(0 \%)$ \\
\hline Well or river & $3(4.0 \%)$ & $0(0 \%)$ & $72(83.7 \%)$ & $80(93.0 \%)$ \\
\hline \multicolumn{5}{|l|}{ Method of human waste disposal† } \\
\hline Indoor plumbing (toilet) & $72(94.7 \%)$ & $64(94.1 \%)$ & $18(20.9 \%)$ & $14(16.3 \%)$ \\
\hline Latrine: open pit & $3(4 \%)$ & $2(2.9 \%)$ & $67(77.9 \%)$ & $71(82.6 \%)$ \\
\hline Latrine: septic tank & $1(1 \%)$ & $2(2.9 \%)$ & $1(1.2 \%)$ & $1(1.2 \%)$ \\
\hline
\end{tabular}

${ }^{\star}$ Estimates for symmetrical numeric variables are given as mean \pm SD.

†Estimates for categorical variables are given as frequency (\%).

produced to visually demonstrate the difference in diarrhoea duration by treatment. The continuous diarrhoea duration data met the proportionality assumption, so Cox proportional hazards models using exact ties and defining the placebo group as the reference were used to produce HRs. An HR>1 indicated that the diarrhoea duration was shorter in the treatment group compared with the placebo group. An analysis of the interaction between treatment and site was tested in the Cox proportional Hazards models and was not significant for any of the pathogen categories (all pathogens, directly targeted pathogens or directly and indirectly targeted pathogens). To assess the impact of PTM202 in a subset of children with prolonged diarrhoea, risk ratios (\% of children with diarrhoea $>\mathrm{X}$ days in the treatment group $/ \%$ of children with diarrhoea $>\mathrm{X}$ days in the placebo group) were calculated for resolution of diarrhoea for days 3 and 7 by study group. Comparison of mean weight gain end points was done using t-tests. SAS V.9.4 (SAS Institute) was used for all data analyses.

A data safety monitoring board was not deemed necessary by the investigators or ethics authorities. An independent study monitor assessed all study procedures at routine site visits throughout the trial. The trial was registered with clinicaltrials.gov, identifier: NCT02385773 (results).

\section{Role of the funding source}

The study funder played a role in the study design and contributed conceptually to the post hoc analysis of outcomes by diarrhoeal pathogen but had no role in data collection, data analysis, data interpretation or writing of this article. The corresponding author had full access to all of the data in the study and had final responsibility for the decision to submit for publication.

\section{RESULTS}

From 9 March 2015 to 25 January 2016, 324 children were enrolled in the study. Details of pre-enrolment screening, consent, randomization, severity arm placement and study event completion are presented in figure 1 .

Of these, $316(97.5 \%)$ children completed the intervention (144 urban children and 172 rural children) and $301(92.9 \%)$ children (135 urban children and 166 rural children) had complete follow-up for the primary end point of diarrhoea duration. Due to the difficulty in enrolling children with severe illness and the subsequent small size of the severe arm, the moderate arm was analysed separately as well as combined with the severe arm subjects. Table 1 displays baseline characteristics for the combined moderate and severe groups enrolled overall, by site and intervention arms.

The study cohort had a mean age of 17.7 months and $46.5 \%$ were female. Mean weight-for-age and weightfor-length were below international averages. No significant differences in medical treatment were noted between the intervention and the placebo arms overall or at any individual site, including prescription of zinc 
Table 2 Comparison of baseline demographic characteristics among Guatemalan children enrolled in PTM202 Diarrhoeal Treatment Trial: urban versus rural study sites

\begin{tabular}{|c|c|c|c|}
\hline Variable & Urban & Rural & $P$ value \\
\hline Age (months) * & $16.5(6.8)$ & $18.7(7.8)$ & 0.007 \\
\hline Height $(\mathrm{cm})$ * & $77.6(7.1)$ & $77.0(6.9)$ & 0.45 \\
\hline Weight at enrolment $(\mathrm{kg})^{*}$ & $9.4(1.7)$ & $8.8(1.6)$ & 0.005 \\
\hline Weight-for-age WHO z-score at enrolment ${ }^{*}$ & $-0.8(1.0)$ & $-1.6(1.1)$ & $<0.0001$ \\
\hline Weight for length at enrolment ${ }^{*}$ & $-0.7(1.0)$ & $-1.2(1.0)$ & $<0.0001$ \\
\hline Estimated per cent dehydration at enrolment ${ }^{\star} \dagger$ & $0.02 \%(3.5 \%)$ & $0.93 \%(4.1 \%)$ & 0.03 \\
\hline \multicolumn{4}{|l|}{ Sex $\neq$} \\
\hline Female & $65(45.0 \%)$ & $82(47.7 \%)$ & 0.73 \\
\hline Male & $79(55.0 \%)$ & $90(52.3 \%)$ & \\
\hline \multicolumn{4}{|l|}{ Intervention $\ddagger$} \\
\hline PTM202 & $76(52.8 \%)$ & $86(50.0 \%)$ & 0.65 \\
\hline Placebo & $68(47.2 \%)$ & $86(50.0 \%)$ & \\
\hline \multicolumn{4}{|l|}{ Water supply to house } \\
\hline Public or bottled water & $135(93.8 \%)$ & $19(11.1 \%)$ & $<0.0001$ \\
\hline Rain water or water tank & $6(4.2 \%)$ & $1(0.6 \%)$ & \\
\hline Well or river & $3(2.1 \%)$ & $152(88.4 \%)$ & \\
\hline \multicolumn{4}{|l|}{ Method of human waste disposalł } \\
\hline Indoor plumbing (toilet) & $136(94.4 \%)$ & $32(18.6 \%)$ & 0.0001 \\
\hline Latrine: open pit & $5(3.5 \%)$ & $138(80.2 \%)$ & \\
\hline Latrine: septic tank & $3(2.1 \%)$ & $2(1.2 \%)$ & \\
\hline Total pathogen count ${ }^{*}$ & $2.7(1.6)$ & $4.8(1.8)$ & $<0.0001$ \\
\hline Total number of directly targeted pathogens ${ }^{\star}$ & $0.5(0.7)$ & $0.9(0.7)$ & $<0.0001$ \\
\hline Total number of directly and indirectly targeted pathogens* & $1.0(0.9)$ & $1.7 \pm 1.0$ & $<0.0001$ \\
\hline Total number of bacteria* & $1.8(1.4)$ & $3.3 \pm 1.4$ & $<0.0001$ \\
\hline Total number of parasites* & $0.1(0.4)$ & $0.7 \pm 0.6$ & $<0.0001$ \\
\hline Total number of viruses* & $0.7(0.7)$ & $0.8 \pm 0.7$ & 0.98 \\
\hline
\end{tabular}

*Estimates for symmetrical numeric variables given as mean \pm SD per case.

†Estimate based on \% weight change from enrolment to 48 hours rehydrated weight.

$\ddagger$ Estimates for categorical variables given as frequency (\%).

( $\mathrm{P}=0.48$ overall, 0.70 urban, 0.63 rural) or antibiotics $(\mathrm{P}=0.18$ overall, 0.41 urban, 0.19 rural $)$.

There were notable differences in demographic and biological characteristics between the urban and rural study populations (table 2 ).

Notably, children enrolled in the rural site were significantly older (18.7 vs 16.5 months; $\mathrm{P}=0.007$ ) and had poorer nutritional status (weight-for-length z-scores -1.6 vs $-0.7 ; \mathrm{P}<0.0001)$. In addition, children at the rural site were much less likely to have a clean supply of drinking water or a sanitary method of human waste disposal. The diarrhoea case pathogen pattern was notably different. The average number of pathogens identified per child in the rural site was almost two times the number in the urban site ( 4.8 vs $2.7 ; \mathrm{P}<0.0001)$. Children in the rural site also had more directly targeted pathogens, indirectly targeted pathogens and both bacterial and parasitic pathogens per stool.
HRs comparing diarrhoea duration in the treatment group and the placebo group are presented in table 3 .

Overall, intervention with PTM202 did not reduce the primary outcome of diarrhoea duration (figure 2). However, the intervention with PTM202 significantly shortened diarrhoea duration in children who had any directly targeted pathogens in the diarrhoeal stool at enrolment ( $\mathrm{P}=0.02)$ (figure 3 ), as well as those who had either directly or indirectly targeted pathogens $(\mathrm{P}=0.04)$. When stratified by site, the impact on diarrhoea duration in children was greatest among children with directly targeted pathogens in the urban site (median reduction in treatment group of 11.9 hours; $\mathrm{P}=0.007$ ) (figure 4). Intervention did not significantly affect diarrhoea duration overall, nor in any pathogen subgroup of children at the rural site (figure 5).

To assess the impact of PTM202 in a subset of children with prolonged diarrhoea, risk ratios were calculated for 
Table 3 Reduction in diarrhoea duration among Guatemalan children treated with PTM202 versus placebo, according to the presence of pathogens targeted directly or indirectly by the nutritional intervention.

\begin{tabular}{|c|c|c|c|c|c|c|}
\hline Pathogen & Intervention & $\mathbf{n}$ & Median hours & HR & $95 \% \mathrm{Cl}$ & $P$ value \\
\hline \multicolumn{7}{|l|}{ All Sites } \\
\hline All pathogens & PTM202 & 154 & 26.7 & 1.10 & 0.88 to 1.39 & 0.40 \\
\hline \multirow[t]{2}{*}{ Directly targeted* } & PTM202 & 78 & 25.5 & 1.46 & 1.07 to 2.00 & 0.02 \\
\hline & Placebo & 85 & 28.7 & & & \\
\hline Directly and indirectly targeted $†$ & Placebo & 119 & 28.7 & & & \\
\hline \multicolumn{7}{|l|}{ Urban } \\
\hline \multirow[t]{2}{*}{ All pathogens } & PTM202 & 71 & 26.4 & 1.30 & 0.93 to 1.83 & 0.13 \\
\hline & Placebo & 64 & 33.5 & & & \\
\hline Directly and Indirectly targeted $†$ & Placebo & 47 & 33.8 & & & \\
\hline \multicolumn{7}{|l|}{ Rural } \\
\hline \multirow[t]{2}{*}{ All pathogens } & PTM202 & 83 & 28.2 & 0.95 & 0.70 to 1.29 & 0.75 \\
\hline & Placebo & 83 & 26.6 & & & \\
\hline \multirow[t]{2}{*}{ Directly targeted* } & PTM202 & 53 & 26.3 & 1.26 & 0.86 to 1.85 & 0.23 \\
\hline & Placebo & 57 & 27.3 & & & \\
\hline \multirow[t]{2}{*}{ Directly and indirectly targeted $†$} & PTM202 & 72 & 28.7 & 1.05 & 0.76 to 1.47 & 0.75 \\
\hline & Placebo & 72 & 27.2 & & & \\
\hline
\end{tabular}

${ }^{*}$ Directly targeted pathogens (enterotoxigenic Escherichia coli, Shiga toxin-positive E. coli, E. coli 0157, Salmonella or Rotavirus) present at baseline.

†Directly or indirectly targeted pathogens (enterotoxigenic Escherichia coli, Shiga toxin-positive E. coli, E. coli 0157, Salmonella, Rotavirus, norovirus or Shigella (enteroinvasive $E$. coli)) present at baseline.

resolution of diarrhoea on days 3 and 7 by study group. Findings in this analysis at day 3 mirrored the overall study findings demonstrating a significant benefit of PTM202 in subjects with one or more targeted organism

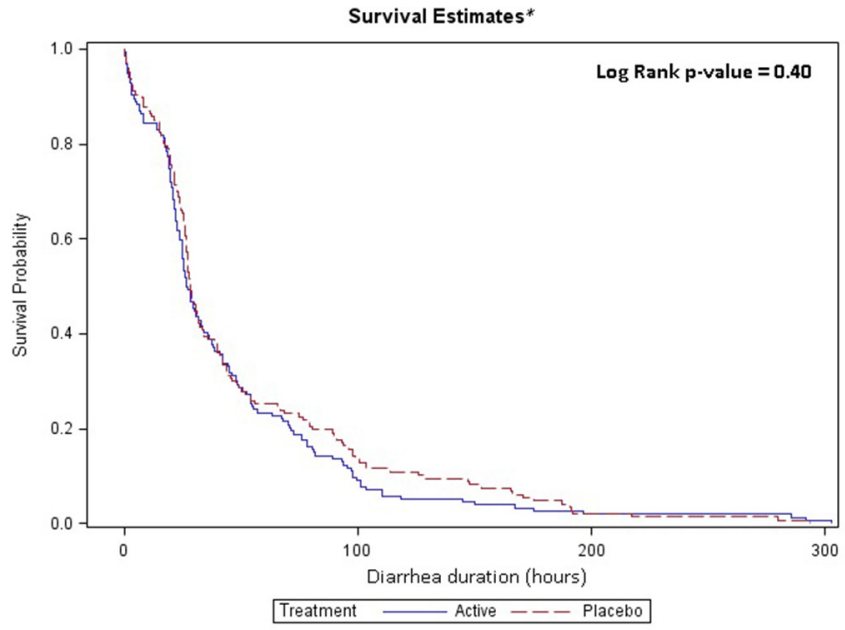

Figure 2 Effect of treatment group for moderate and severe patients with any pathogen at the urban andrural sites combined. *154 patients were treated with P2M202 and 147 subjects were treated with placebo. at the urban site (96\% resolution vs $71.4 \%$; risk ratio: 1.34; $\mathrm{P}=0.026)$ and no measurable impact in subjects at the rural site or when all pathogens were considered. At

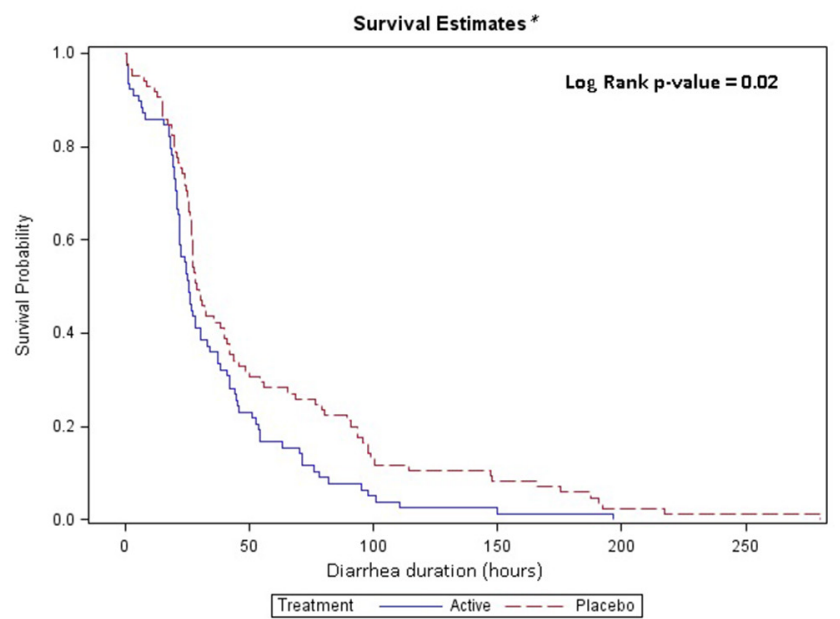

Figure 3 Effect of treatment group for moderate and severe patients with any directly targeted pathogenat the urban and rural sites combined. *78 subjects were treated with P2M202 and 85 subjects were treated with placebo. 


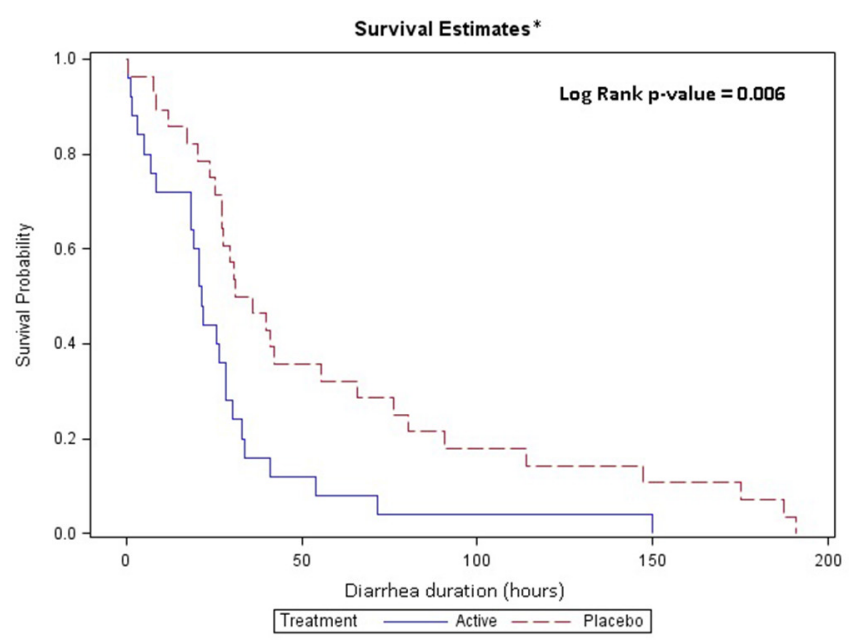

Figure 4 Effect of treatment group for moderate and severe patients with any directly targeted pathogenat the urban site. ${ }^{\star} 25$ subjects were treated with P2M202 and 28 subjects were treated with placebo.

day 7, a similar pattern was observed but did not demonstrate statistical significance in children with from the urban site with directly targeted pathogens $(\mathrm{P}=0.092)$.

Given the significant association between PTM202 administration and shortened diarrhoea duration in children with any directly targeted pathogen at the urban study site, we examined the effect of the intervention on diarrhoea duration by individual pathogen. In urban children, PTM202 was associated with significant reductions in diarrhoeal duration in children with STEC (a directly targeted pathogen) as well as Clostridium difficile or astrovirus (table 4).

However, numbers of subjects with the latter organisms not specifically targeted by PTM202 were very low (astrovirus $\mathrm{n}=6$; $C$. difficile $\mathrm{n}=12$ ).

An examination of the effect of PTM202 intervention on weight gain 2 and 4 weeks post-treatment initiation in

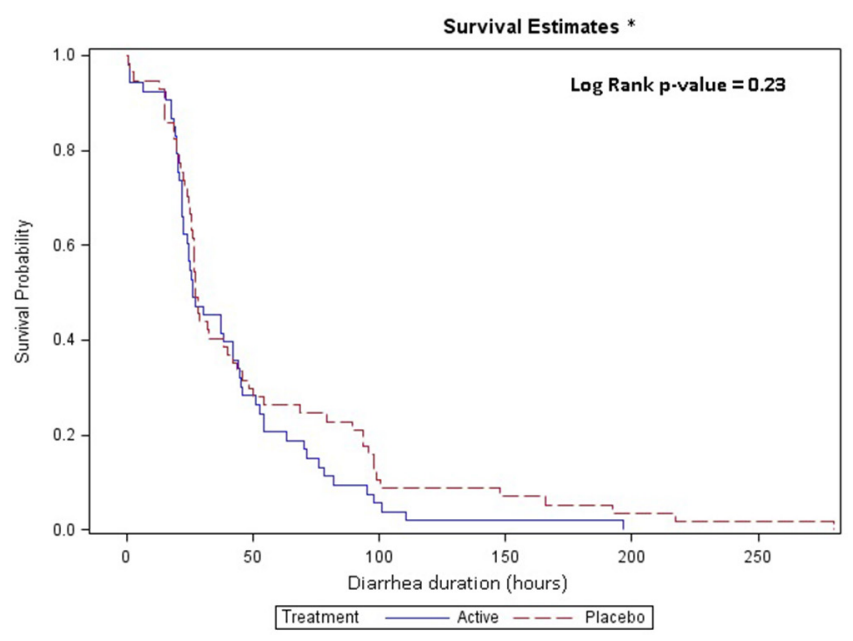

Figure 5 Effect of treatment for moderate patients with any directly targeted organism at the rural site. ${ }^{*} 53$ subjects were treated with P2M202 and 57 subjects were treated with placebo. subjects who had a completed diarrhoea outcome did not show any significant improvements in weight gain in the intervention group compared with the placebo group in the study population overall, nor in analysis stratified by site or presence of PTM202-targeted organisms at enrolment.

PTM202 was found to be very safe, and no adverse or SAEs were associated with study interventions. All adverse events were common or expected illnesses, injuries or other medical conditions (online supplementary table). There was no statistical difference in the frequency of SAEs in aggregate between the PTM202 and the placebo arms (PTM202: 5/165 vs placebo 2/159; $\mathrm{P}=0.45$ ).

\section{DISCUSSION}

In a randomised, double-blind, placebo-controlled trial among Guatemalan children with acute diarrhoea, an impact of the nutrition-based intervention PTM202 on diarrhoeal duration was not noted for all subjects combined, but led to a median reduction of approximately 12 hours in an urban subgroup of children whose diarrhoea was associated with the presence of one or more specifically targeted pathogens in their stool at the time of enrolment.

The concepts underlying the development of PTM202, which combines both non-specific colostrum-based factors that may promote gut health and postinfectious recovery with animal-derived, pathogen-specific antibodies, and the rationale for efficacy as a therapeutic agent in acute diarrhoea are based on a significant body of clinical evidence. Breastfeeding is strongly recommended for children with diarrhoea who can tolerate oral intake, and breast milk contains factors including secretory immunoglobulins, fatty acids, lactoferrin, glycoconjugates, oligosacharrides, prebiotics and immune modulators, which can prevent and ameliorate infectious diarrhoea, provide nutrition, promote healing of the gut and restore healthy microbiota. ${ }^{1415}$ Many of the potentially beneficial components of breast milk may be found in bovine colostrum. ${ }^{16}$ The therapeutic applications of bovine colostrum have been reviewed by Struff $e t a l$ and more recently by Rathe $e t a l$, and are characterised by heterogeneous methodologies and results across a spectrum of clinical conditions in both adult and paediatric populations. ${ }^{10} 17$ Treatment with bovine colostrum containing high titres against human rotavirus led to improvements in stool output, fewer treatment requirements and virus neutralisation in two randomised controlled trials and one non-randomised trial in paediatric patients with rotavirus diarrhoea. ${ }^{18-20}$ However, one randomised controlled trial failed to demonstrate any clinical impact in 135 Finnish children. ${ }^{21}$ Rotavirus hyperimmune colostrum has also shown potential effect as a preventative treatment for diarrhoea both in hospital and community settings. ${ }^{22} 23$ The impact of bovine colostrum on paediatric bacterial enteritis has not been clearly demonstrated; Huppertz 
Table 4 Effect of PTM202 on duration of diarrhoea among Guatemalan children by the presence of individual pathogen at urban site enrolment

\begin{tabular}{|c|c|c|c|c|c|c|}
\hline \multirow[b]{2}{*}{ Pathogen } & \multirow[b]{2}{*}{ Intervention } & \multirow[b]{2}{*}{$\mathbf{n}$} & \multirow{2}{*}{$\begin{array}{l}\text { Median } \\
\text { hours }\end{array}$} & \multirow[b]{2}{*}{ HR } & \multirow[b]{2}{*}{$95 \% \mathrm{Cl}$} & \multirow[b]{2}{*}{$P$ value } \\
\hline & & & & & & \\
\hline \multirow[t]{2}{*}{ ETEC $†$} & PTM202 & 16 & 27.0 & 1.86 & 0.93 to 3.70 & 0.08 \\
\hline & Placebo & 21 & 35.8 & & & \\
\hline \multirow[t]{2}{*}{ STEC/0157† } & PTM202 & 5 & 3.0 & 12.17 & 1.38 to 107.84 & 0.025 \\
\hline & Placebo & 6 & 28.9 & & & \\
\hline \multirow[t]{2}{*}{ Rotavirus $†$} & PTM202 & 6 & 20.1 & 1.38 & 0.41 to 4.67 & 0.60 \\
\hline & Placebo & 6 & 41.5 & & & \\
\hline \multirow[t]{2}{*}{ Salmonella† } & PTM202 & 1 & $\mathrm{~N} / \mathrm{A}$ & 2.45 & 0.15 to 39.72 & 0.53 \\
\hline & Placebo & 3 & 41.9 & & & \\
\hline \multirow[t]{2}{*}{ Norovirusł } & PTM202 & 26 & 24.6 & 1.66 & 0.94 to 2.94 & 0.08 \\
\hline & Placebo & 27 & 30.9 & & & \\
\hline \multirow[t]{2}{*}{ Shigella (EIEC)‡ } & PTM202 & 14 & 25.6 & 2.52 & 0.88 to 7.23 & 0.09 \\
\hline & Placebo & 7 & 41.1 & & & \\
\hline \multirow[t]{2}{*}{ Campylobacter } & PTM202 & 16 & 25.0 & 1.67 & 0.74 to 3.78 & 0.22 \\
\hline & Placebo & 11 & 39.8 & & & \\
\hline \multirow[t]{2}{*}{ Clostridium difficile } & PTM202 & 5 & 22.6 & 6.43 & 1.17 to 35.29 & 0.03 \\
\hline & Placebo & 7 & 30.9 & & & \\
\hline \multirow[t]{2}{*}{ Plesiomonas } & PTM202 & 1 & $\mathrm{~N} / \mathrm{A}$ & $\mathrm{N} / \mathrm{A}$ & $\mathrm{N} / \mathrm{A}$ & N/A \\
\hline & Placebo & 1 & N/A & & & \\
\hline \multirow{2}{*}{$\begin{array}{l}\text { Enteroaggregative } \\
\text { Escherichia coli }\end{array}$} & PTM202 & 33 & 25.6 & 1.15 & 0.71 to 1.89 & 0.57 \\
\hline & Placebo & 32 & 30.1 & & & \\
\hline \multirow[t]{2}{*}{ Enteropathogenic E. coli } & PTM202 & 27 & 25.6 & 1.63 & 0.94 to 2.83 & 0.08 \\
\hline & Placebo & 29 & 30.9 & & & \\
\hline \multirow[t]{2}{*}{ Cryptosporidium } & PTM202 & 3 & 19.0 & 1.70 & 0.34 to 8.62 & 0.52 \\
\hline & Placebo & 4 & 33.9 & & & \\
\hline \multirow[t]{2}{*}{ Giardia } & PTM202 & 7 & 5.2 & 2.27 & 0.47 to 11.04 & 0.31 \\
\hline & Placebo & 3 & 17.2 & & & \\
\hline \multirow[t]{2}{*}{ Adenovirus } & PTM202 & 8 & 23.4 & 1.53 & 0.52 to 4.50 & 0.44 \\
\hline & Placebo & 7 & 65.7 & & & \\
\hline \multirow[t]{2}{*}{ Astrovirus* } & PTM202 & 3 & 22.6 & 2.09 & 1.57 to 2.79 & $<0.0001$ \\
\hline & Placebo & 3 & 46.3 & & & \\
\hline \multirow[t]{2}{*}{ Sapovirus } & PTM202 & 7 & 35.8 & 0.89 & 0.31 to 2.55 & 0.83 \\
\hline & Placebo & 9 & 41.4 & & & \\
\hline
\end{tabular}

*HR not calculable due to bimodal distribution of the data; risk ratio using Poisson distribution is presented.

†Organism directly targeted by PTM202.

‡Organism indirectly targeted by PTM202.

EIEC, enteroinvasive E.coli; ETEC, enterotoxigenic E.coli.

et alfound a reduced stool frequency in thirteen German patients with E. coli enteritis, but no impact of hyperimmune colostrum was observed in Bangladeshi children with enteropathogenic E. coli or ETEC associated diarrhoea, nor in children infected with Shigella. ${ }^{24-26}$

Another nutritional source of potential protective immunoglobulin is hen's egg yolk containing IgY, and therapeutic mechanisms, animal models and clinical trials were recently reviewed by Thu et al. ${ }^{27}$ Levels of egg
IgY specific for human pathogens, including rotavirus, can be augmented by vaccination of hens and rotavirus hyperimmune egg yolk reduced diarrhoea duration and treatment requirements in one clinical trial involving 54 rotavirus infected children in Myanmar. ${ }^{28}$

Despite numerous trials suggesting short-term benefit of colostrum or hen egg-derived nutritional therapeutics, the ability to extrapolate results to a broad clinical application is limited by low numbers of subjects, 
heterogeneous study populations and a focus on single pathogens. In our trial, the study design (randomised, placebo-controlled, adequately powered study, in a diverse population in two distinct settings) may permit a clearer understanding of the potential impact of nutrition-based diarrhoeal therapeutics.

Identification of stool pathogens by PCR is a particular strength of this study, which allowed us to observe an impact of PTM202 in Guatemalan children with targeted organisms and describe the etiological characteristics of subjects for whom treatment did not show benefit. This methodology has significant advantages over therapeutic trials in which diagnostics target only a single pathogen because the etiologies of diarrhoea, particularly in LMICs, are myriad and complex. Coinfection, chronic pathogen carriage and reinfection are common, as demonstrated by our data as well as two recent major efforts to characterise stool diarrhoeal pathogens in low-income paediatric populations. ${ }^{29} 30$ At the same time, it is important to acknowledge that this methodology and the complexity of the stool findings in our study also present significant challenges in analysis of any potential therapeutic effect of PTM202 by aetiology, and indeed to assign any specific aetiology among many as the cause of the diarrhoea episode. Although we initially planned subanalysis by individual targeted organisms, our subsequent post hoc decision to analyse outcomes in a subgroup of patients characterised by the presence of at least one targeted organism was driven in large part by the fact that only $11 \%$ of our subjects had a single pathogen at enrolment.

In addition to these analytical challenges, there are other limitations to note. Prominent among these was the lack of subjects in the severe arm, which was a reflection of very low numbers of children hospitalised for diarrhoeal disease overall. As a consequence, we have been unable to more definitively assess trial outcomes in children with more severe illness. Additionally, though stool consistency can be another measure of response to diarrhoeal treatment and parental description of stool consistency was sought via diary card through the trial period, these reports were incomplete and inconsistent. We were thus unable to analyse any impact of PTM202 on stool consistency beyond the primary outcome, which was focused on resolution of watery diarrhoea leading to fluid, electrolyte and micronutrient loss, most significant in this population. Finally, although we included two distinct Guatemalan populations, the efficacy of PTM202 may differ in populations in other regions of the world, where there may be critical differences in infectious exposure and diarrhoeal aetiology, underlying malnutrition and gut inflammation.

Two observations from this trial are of particular importance in addressing the potential role for PTM202 in the treatment of childhood diarrhoea in LMICs. First, overall efficacy was only demonstrated in children with at least one targeted (direct or indirect) pathogen. This observation supports a direct effect of specific antibody in PTM202 (either alone or through a synergistic mechanism with non-specific elements of the product) and suggests that the most effective use of PTM202 may be in cases where a targeted pathogen can be rapidly identified through diagnostic testing, or when a targeted pathogen is highly likely based on local epidemiology (eg, an outbreak scenario). Furthermore, the potential capability suggested in our trial to specifically target individual pathogens through PTM202 design may open the door to future development to target either a broader spectrum of etiologies (resulting in more universal utility) or specific individual pathogens associated with disease outbreaks.

The second key observation is that the reduction in diarrhoeal duration from PTM202 in patients with targeted organisms was driven primarily by the benefit observed in subjects in the urban area. Guatemala City is typical of large urban centres in many middle-income emerging economies, where improved water and hygiene infrastructure are more universal, and where despite widespread poverty, food access is not as critical as it is in many rural low-income areas of the world. Conversely, the rural site is very isolated and lacking in hygiene infrastructure, and prior needs assessments performed by our institution have demonstrated that food insecurity is acute. Additionally, there is regular flooding throughout the rainy season, exacerbating human waste exposure. These factors are clearly demonstrated in socioeconomic and environmental data for enrolled subjects. A result of these environmental disparities is a notable difference in the number and spectrum of diarrhoeal pathogens. We hypothesise that in addition to the direct impact on diarrhoeal duration from numerous non-targeted pathogens in the rural population, this infectious burden was contributing to an inflammatory enteropathy that may have overwhelmed any potential response to the therapeutic intervention.

These observations suggest how PTM202 may complement other currently recommended treatments for diarrhoea, most notably zinc. Zinc supplementation is recommended by the WHO for children in low-income countries with acute diarrhoea, and appears most efficacious in younger infants and in children at high risk of zinc deficiency. ${ }^{6}$ Zinc is an important cofactor in mucosal immune defense and recovery from diarrhoeal illness. In our rural study population, the higher likelihood of zinc deficiency and the high overall infectious burden may have contributed to the lack of impact of PTM202, even in subjects with targeted organisms. In contrast, zinc deficiency is likely to be less common in an urban Guatemalan population, and this phenomenon combined with the lower infectious burden may have permitted the better efficacy of PTM202 in this population.

The ability to target diarrhoea caused by specific pathogens also suggest the tantalising possibility that PTM202 could replace treatment with antibiotics in some cases of non-bloody bacterial diarrhoea. The use of antibiotics for acute infectious diarrhoea is extremely common in 
LMICs, despite recommendations that they not be used for non-bloody diarrhoea and observations that this practice may increase the risk of some complications (eg, haemolytic uremic syndrome in patients with STEC), prolong shedding of certain etiologies including Salmonella, alter the intestinal microbiome and, most importantly, contribute to the global problem of antimicrobial resistance. ${ }^{7}$ Assessing the role of PTM202 in reducing antibiotic use for acute non-bloody diarrhoea in LMICs should be an important area of future research.

The results of this trial raise a number of additional questions for future research. Notably, a post hoc analysis of potential efficacy of PTM202 at 24 and 48 hours after initial treatment ingestion (proportion of subjects with resolved diarrhoea at 24 hours: $56.0 \%$ treatment vs $25.0 \%$ placebo at 24 hours, $\mathrm{P}=0.021$; at 48 hours: $88.0 \%$ treatment vs $64.3 ; \mathrm{P}=0.045$ ) suggests that the observed impact of the PTM202 in patients with targeted organisms may not require three doses. Future study will address this critical question directly. Additional potential questions include utility of PTM202 in combination with other diarrhoea therapies (particularly zinc) and potential use of a similar intervention as a preventative treatment in outbreak scenarios or for travellers to high-risk areas. It will also be important to continue to define the optimal target patient population for PTM202 treatment of acute diarrhoea and determine if the efficacy noted here can be duplicated in populations from other global regions. Finally, as the intervention is based on globally available food sources, the potential for local manufacture may lead to lower long-term costs and facilitate broader availability.

\section{CONCLUSION}

In this randomised, double-blind, placebo-controlled trial, a 3-day course of PTM202-a novel, safe, nutrition-based intervention consisting of cow's milk colostrum and immune-enhanced hen's egg-shortened acute diarrhoeal duration among urban Guatemalan children with specifically targeted pathogens in stool. The results suggest that PTM202 may represent an additional therapeutic tool for the intervention of childhood diarrhoea in paediatric populations with similar underlying demographics and stool pathogen distribution.

\author{
Author affiliations \\ ${ }^{1}$ Center for Global Health, Colorado School of Public Health, Aurora, Colorado, USA \\ 2Department of Epidemiology, Colorado School of Public Health, Aurora, Colorado, \\ USA \\ ${ }^{3}$ Pediatric Infectious Disease, Hospital Roosevelt, Guatemala City, Guatemala \\ ${ }^{4}$ Centro de Estudios en Salud, Universidad de Valle de Guatemala, Guatemala City, \\ Guatemala, USA \\ ${ }^{5}$ Department of Pediatrics, University of Colorado School of Medicine, Aurora, \\ Colorado, USA \\ ${ }^{6}$ Department of Pathology and Laboratory Medicine, Children's Hospital Colorado, \\ Aurora, Colorado, USA
}

Acknowledgements The authors would like to acknowledge and express their gratitude to all study participants and their families for participating in this trial. They would also like to thank Mirsa Ariano for administrative support, Jose David
Miguel Mendoza and Jose Guillermo Rivera for the management of a complex data set, Alma Zacarias and Maria Alejandra Paniagua-Avila for site coordination in rural Guatemala, BioFire for kindly providing FilmArray stool diagnostic kits at cost to our laboratory, Maria Renee Lopez for laboratory/specimen oversight in Guatemala and Joshua Lechner, Kristin Pretty and Darcy Velasquez at the Children's Hospital Colorado Microbiology Laboratory for processing stool samples so efficiently and professionally.

Contributors JTG, MAM, EJA and SB designed and executed the study and interpreted the data. MAM, DMC and ILC-R coordinated study participant recruitment and treatment. Data analysis was conducted by JTG, MML and SB. Laboratory analysis and interpretation was coordinated by SRD and CCR. Preparation of the report was performed by JTG, MML and SB.

Funding This study was funded by PanTheryx, Boulder, USA, the manufacturer of PTM202.

Competing interests None declared.

Ethics approval Colorado Multiple Institutional Review Board, and in Guatemala from the National Committee for Ethics in Health, Ministry of Public Health and Social Assistance, Hospital Roosevelt Ethics Committee and the Universidad del Valle de Guatemala Ethics Committee.

Provenance and peer review Not commissioned; externally peer reviewed.

Data sharing statement We are committed to providing open access to our data set through a research data depository. We anticipate use of Dryad for this service and have initiated an account with Dryad for this purpose.

Open Access This is an Open Access article distributed in accordance with the Creative Commons Attribution Non Commercial (CC BY-NC 4.0) license, which permits others to distribute, remix, adapt, build upon this work non-commercially, and license their derivative works on different terms, provided the original work is properly cited and the use is non-commercial. See: http://creativecommons.org/ licenses/by-nc/4.0/

(c) Article author(s) (or their employer(s) unless otherwise stated in the text of the article) 2017. All rights reserved. No commercial use is permitted unless otherwise expressly granted.

\section{REFERENCES}

1. Liu L, Johnson HL, Cousens S, et al. Global, regional, and national causes of child mortality: an updated systematic analysis for 2010 with time trends since 2000. Lancet 2012;379:2151-61.

2. WHO. Diarrhoeal disease. 2013 http://www.who.int/mediacentre/ factsheets/fs330/en/ (accessed 5 Apl 2017).

3. Richard SA, Black RE, Gilman RH, et al. Diarrhea in early childhood: short-term association with weight and long-term association with length. Am J Epidemiol 2013;178:1129-38.

4. Black RE, Allen LH, Bhutta ZA, et al. Maternal and child undernutrition: global and regional exposures and health consequences. Lancet 2008;371:243-60.

5. UNICEF/WHO. Joint statement: clinical management of acute diarrhoea. 2004. 8 http://www.who.int/maternal_child_adolescent/ documents/who fch cah 04 7/en/

6. Lazzerini $\mathrm{M}$, Wanzira $\mathrm{H}$. Oral zinc for treating diarrhoea in children. Cochrane Database Syst Rev 2016;12:CD005436.

7. Rogawski ET, Platts-Mills JA, Seidman JC, et al. Use of antibiotics in children younger than two years in eight countries: a prospective cohort study. Bull World Health Organ 2017;95:49-61.

8. Enos MK, Burton JP, Dols J, et al. Probiotics and nutrients for the first 1000 days of life in the developing world. Benef Microbes 2013;4:3-16.

9. Guarino A, Dupont C, Gorelov AV, et al. The management of acute diarrhea in children in developed and developing areas: from evidence base to clinical practice. Expert Opin Pharmacother 2012;13:17-26.

10. Rathe M, Müller K, Sangild PT, et al. Clinical applications of bovine colostrum therapy: a systematic review. Nutr Rev 2014;72:237-54.

11. Yolken RH, Leister F, Wee SB, et al. Antibodies to rotaviruses in chickens' eggs: a potential source of antiviral immunoglobulins suitable for human consumption. Pediatrics 1988;81:291-5.

12. Hoare S, Poppitt SD, Prentice AM, et al. Dietary supplementation and rapid catch-up growth after acute diarrhoea in childhood. $\mathrm{Br} \mathrm{J}$ Nutr 1996;76:479-90.

13. Dinleyici EC, Vandenplas Y. PROBAGE Study Group. Lactobacillus reuteri DSM 17938 effectively reduces the duration of acute diarrhoea in hospitalised children. Acta Paediatr 2014;103:e300-5. 
14. Newburg DS, Peterson JA, Ruiz-Palacios GM, et al. Role of human-milk lactadherin in protection against symptomatic rotavirus infection. Lancet 1998;351:1160-4.

15. Ballard O, Morrow AL. Human milk composition: nutrients and bioactive factors. Pediatr Clin North Am 2013;60:49-74.

16. Gopal PK, Gill HS. Oligosaccharides and glycoconjugates in bovine milk and colostrum. Br J Nutr 2000;84(Suppl 1):S69-74.

17. Struff WG, Sprotte G. Bovine colostrum as a biologic in clinical medicine: a review--Part II: clinical studies. Int J Clin Pharmacol Ther 2008:46:211-25.

18. Sarker SA, Casswall TH, Mahalanabis D, et al. Successful treatment of rotavirus diarrhea in children with immunoglobulin from immunized bovine colostrum. Pediatr Infect Dis $J$ 1998;17:1149-54.

19. Mitra AK, Mahalanabis D, Ashraf $\mathrm{H}$, et al. Hyperimmune cow colostrum reduces diarrhoea due to rotavirus: a double-blind controlled clinical trial. Acta Paediatr 1995;84:996-1001.

20. Hilpert $\mathrm{H}$, Brüssow $\mathrm{H}$, Mietens $\mathrm{C}$, et al. Use of bovine milk concentrate containing antibody to rotavirus to treat rotavirus gastroenteritis in infants. J Infect Dis 1987;156:158-66.

21. Ylitalo S, Uhari M, Rasi S, et al. Rotaviral antibodies in the treatment of acute rotaviral gastroenteritis. Acta Paediatr 1998;87:264-7.

22. Patel K, Rana R. Pedimune in recurrent respiratory infection and diarrhoea--the Indian experience--the pride study. Indian J Pediatr 2006;73:585-91.

23. Davidson GP. Passive protection against diarrheal disease. J Pediatr Gastroenterol Nutr 1996;23:207-12.
24. Ashraf $\mathrm{H}$, Mahalanabis D, Mitra AK, et al. Hyperimmune bovine colostrum in the treatment of shigellosis in children: a double-blind, randomized, controlled trial. Acta Paediatr 2001;90:1373-8.

25. Casswall TH, Sarker SA, Faruque SM, et al. Treatment of enterotoxigenic and enteropathogenic Escherichia coli-induced diarrhoea in children with bovine immunoglobulin milk concentrate from hyperimmunized cows: a double-blind, placebo-controlled, clinical trial. Scand J Gastroenterol 2000;35:711-8.

26. Huppertz HI, Rutkowski S, Busch DH, et al. Bovine colostrum ameliorates diarrhea in infection with diarrheagenic Escherichia coli, shiga toxin-producing E. Coli, and E. coli expressing intimin and hemolysin. J Pediatr Gastroenterol Nutr 1999;29:452-6.

27. Thu HM, Myat TW, Win MM, et al. Chicken egg yolk antibodies (igy) for prophylaxis and treatment of rotavirus diarrhea in human and animal neonates: a concise review. Korean J Food Sci Anim Resour 2017;37:1-9.

28. Rahman S, Higo-Moriguchi K, Htun KW, et al. Randomized placebocontrolled clinical trial of immunoglobulin $Y$ as adjunct to standard supportive therapy for rotavirus-associated diarrhea among pediatric patients. Vaccine 2012;30:4661-9.

29. Kotloff KL, Nataro JP, Blackwelder WC, et al. Burden and aetiology of diarrhoeal disease in infants and young children in developing countries (the Global Enteric Multicenter Study, GEMS): prospective, case-control study. Lancet 2013;382:209-22.

30. Platts-Mills JA, Babji S, Bodhidatta L, et al. Pathogen-specific burdens of community diarrhoea in developing countries: a multisite birth cohort study (MAL-ED). Lancet Glob Health 2015;3:e564-e575. 